\title{
The Fate of Abstracts Presented at the Annual Meeting of the Congress of Neurologic Surgeons
}

\author{
Cameron A. Elliott'1, Vivek Mehta1, Candice Poon², Monika Oliver³, Sita Gourishankar4 \\ ${ }^{1}$ Division of Neurosurgery, Department of Surgery, University of Alberta, Edmonton, Canada \\ ${ }^{2}$ Division of Neurosurgery, Department of Surgery, University of Calgary, Calgary, Canada \\ ${ }^{3}$ Faculty of Science, University of Alberta, Edmonton, Canada \\ ${ }^{4}$ Division of Nephrology and Transplant Immunology, Department of Medicine, University of Alberta, \\ Edmonton, Canada \\ Email: sitag@ualberta.ca
}

Received 9 October 2015; accepted 3 January 2016; published 6 January 2016

Copyright (C) 2016 by authors and Scientific Research Publishing Inc.

This work is licensed under the Creative Commons Attribution International License (CC BY). http://creativecommons.org/licenses/by/4.0/

(c) (i) Open Access

\begin{abstract}
The purpose of this study was to determine the publication rate and time to publication following abstract presentation at a major neurosurgical meeting, namely the 2005 annual meeting of the Congress of Neurological Surgeons (CNS). We reviewed all 754 abstracts presented at the 2005 CNS annual meeting to determine publication status and identify factors that are associated with publication. A standardized database search was conducted to determine publication status. Predictors of publication were examined. Of the 754 abstracts presented, $383(50.8 \%)$ were published after 5.3 years. The median time to publication was 450 days (range-646 to 1953 days) with $16 \%$ being published prior to the meeting. Oral presentations had a publication rate of $64.0 \%$ compared to $48.3 \%$ for poster presentations. Of the abstracts not published prior to the meeting, $77 \%$ were published within 3 years of the meeting. In univariate analysis, neuro-oncology abstracts (OR 1.88; 95\% CI 1.34 - 2.64; $p<0.001)$ and abstracts selected for oral presentation (OR $1.97 ; 95 \%$ CI $1.30-2.99 ; p=0.001$ ) had a significantly higher likelihood of publication. These associations remained independent in multivariate analysis. Neuro-oncology abstracts $(p=0.004)$, basic science research $(p=0.003)$ and abstracts selected for oral presentation $(p=0.036)$ were published in higher impact factor journals. Half of presentations at the annual CNS meeting will be published in peer-reviewed journals with a median time to publication of 450 days. Neuro-oncology abstracts and those abstracts selected for oral presentation have a significantly higher likelihood of publication.
\end{abstract}

\section{Keywords}

CNS, Impact Factor, Publication Rate 


\section{Introduction}

Attending and presenting abstracts at prominent neurosurgical meetings is an exciting and interactive opportunity to disseminate new ideas. However, these meeting abstracts may be incomplete summaries of preliminary data, for which access is effectively limited to those in attendance at the meeting and which are subject to an abbreviated, albeit, competitive peer-review process. Abstract presentation may influence clinical practice of clinicians in attendance but are not indexed in frequently used electronic databases. Therefore, abstracts which do not ultimately and in a timely fashion culminate in full-text publication may have a minimal impact on the neurosurgical community in general.

The publication rate following abstract presentation is one accepted indicator of conference quality. In nonneurosurgical fields the publication rate following abstract presentation ranges from $8 \%-84 \%$ [1]-[5]. Achieving full-text publication can be a frustrating endeavor especially given the rigors of clinical practice. In an attempt to better understand this challenge one can investigate which factors determine successful publication. Factors predictive of success in other fields include acceptance for conference presentation, larger sample size, selection for oral presentation, basic science research, industry sponsorship and positive results [2] [4]-[10]. In order to examine these issues in neurosurgery we conducted a descriptive, observational, retrospective study to determine the publication rate and time to publication following abstract presentation at the $55^{\text {th }}$ annual meeting of the Congress of Neurological Surgeons (CNS) held in Boston, Massachusetts October 8 - 13, 2005. Our secondary objective was to identify factors predictive of subsequent full-text publication following abstract presentation and factors predictive of publication in higher impact journals.

\section{Materials and Methods}

\subsection{Eligible Abstracts}

We reviewed all accepted and presented abstracts $(n=754)$ from the online abstract archive (http://w3.cns.org/university/abstracts/index2.asp) for the $55^{\text {th }}$ annual meeting of the Congress of Neurological Surgeons (CNS) held October 8 - 13, 2005, in Boston, Massachusetts. Withdrawn (accepted, but not presented; $\mathrm{n}=1)$ or duplicate abstracts $(\mathrm{n}=1)$ were excluded.

\subsection{Data Abstraction}

Data extracted through abstract review included: neurosurgical subspecialty (vascular, neuro-oncology, spine, functional, peripheral nerve, neurotrauma, pediatrics, radiosurgery, general), country of origin of principal author, presentation type (oral vs. poster), research type (clinical vs. basic science), study design (according to epidemiological definitions), sample size, presence of statistically significant results (defined as $p<0.05$ ), new technology or surgical technique, industry sponsorship and $\mathrm{PhD}$ listed by the principal investigator. Data abstraction was carried out by a single investigator and validated by a second investigator who reviewed randomly selected abstracts. Inconsistencies were resolved through consensus.

\subsection{Identifying Full-Text Publications}

Similar to previous studies in non-neurosurgical fields publication status was determined using computerized database searches conducted independently by two investigators in MEDLINE, Scopus and Google Scholar [2] [10]. The first, second, corresponding and final author as well as abstract keywords were searched individually and combined. Following the initial search of all three databases by one investigator, an alternate investigator undertook a crossover search of abstracts not yielding a publication. A match between the identified publication and the abstract in question was determined by comparison of the title, author(s), objectives, methodology and results to discern whether the publication was judged to be a direct result of abstract content. If no match was found in all three databases by two investigators the abstract was considered unpublished. The time to publication was defined as the difference in days between the date of publication (rounded to the first day of the month if day not specified or listed as January $1^{\text {st }}$ if month not specified) and the first day of the conference (October 8, 2005). The publication search was carried out between January 1, 2011 and April 30, 2011.

\subsection{Impact Factor Analysis}

The ISI Web of Knowledge citation database (Philadelphia, USA) was used to determine the 2010 impact factor 
for each journal. Publications in journals not indexed or in journals that did not have an impact factor listed were excluded from the analysis.

\subsection{Statistical Analysis}

Categorical variables were compared using chi-square test. Continuous variables were reported as means with standard deviations or in the case of outliers as medians. Continuous variables were compared using the $t$ test. To determine predictors of full-text publication, odds ratios and their corresponding 95\% confidence intervals were calculated using univariate and multivariate logistic regression analysis. All statistical analyses were performed by using SPSS (version 18.0 for Windows).

\section{Results}

At the $55^{\text {th }}$ annual meeting of the CNS, 1134 abstracts were submitted for presentation, of which 755 were ultimately selected for presentation (personal communication, CNS). Of these 755 abstracts, which are available at the CNS online abstract archive (http://w3.cns.org/university/abstracts/index2.asp), one was withdrawn prior to the conference, resulting in 754 abstracts being included in this analysis.

\subsection{Abstract Characteristics}

Abstract characteristics are outlined in Table 1. Of the abstracts presented, only 114 (15.1\%) were chosen for oral presentation while 638 (84.9\%) were poster presentations. Clinical research made up 79.7\% (601) of abstracts while basic science research made up $20.3 \%$ (153). The top three neurosurgical subspecialty areas represented were neuro-oncology (24\%), spine (20\%) and vascular (18\%). The vast majority of abstracts originated from the United States (534, 70.8\%). A new technology, surgical technique or drug was mentioned in 278 (36.9\%) of abstracts. Statistically significant results were mentioned in 135 (17.9\%) of abstracts although it was difficult to reliably determine whether these related to primary outcome due to a paucity of detail in many abstracts. The principal author held a $\mathrm{PhD}$ in $158(21.0 \%)$ of abstracts accepted for presentation. Amongst clinical research, cohort studies (50.5\%) and case series (16.6\%) were the most common study design while only $2.3 \%$ were randomized-controlled trials.

\subsection{Publication Rate and Time to Publication}

Of the 754 abstracts presented at the 2005 CNS annual meeting 383 (50.8\%) resulted in full-text publications at 5.3 years. Oral presentations had a publication rate of $64.0 \%$ (73/114) compared to $48.3 \%$ (308/638) for poster presentations. Interestingly, $16.2 \%$ (61) of abstracts were published prior to the meeting with $1.3 \%$ being published in the year prior to the conference (2004) before the abstract deadline. Of the articles that were not published prior to the meeting, most were published within two (57\%) or three (77\%) years, with a median time to publication of 450 days with a wide range; the earliest publication occurring 646 days prior to the conference and the latest occurring 1953 days after the conference (Figure 1).

\subsection{Journal of Publication and Impact Factor}

Articles were published in 121 different journals with 236 (61.4\%) of publications in dedicated neurosurgery journals and one-third of publication occurring in either Neurosurgery or the Journal of Neurosurgery. The top 8 journals in which publications occurred and their 2010 impact factors are listed in Table 2. The overall mean impact factor for all publications was 3.319 (0.309 - 53.484). The mean impact factor for publication in dedicated neurosurgery journals was 2.75 compared to a mean impact factor of 3.80 for publications in all other journals.

\subsection{Univariate Predictors of Publication}

Neuro-oncology abstracts had a significantly higher likelihood of publication with an odds ratio of 1.88 (95\% CI 1.34 - 2.64; $\mathrm{p}<0.001$ ) relative to all other abstracts. Abstracts accepted for an oral presentation were also more frequently published compared to poster presentations (OR 1.91; 95\% CI $1.26-2.88, \mathrm{p}=0.002$ ). Although there was no statistically significant difference in publication rate between basic and clinical research, basic science 
Table 1. Abstract characteristics.

\begin{tabular}{|c|c|c|}
\hline Characteristic & n (\%) & Published, n (\%) \\
\hline Number of Abstracts & 754 & $383(50.8)$ \\
\hline \multicolumn{3}{|l|}{ Presentation type } \\
\hline Oral presentation & $114(15.2)$ & $73(64.0)$ \\
\hline Poster presentation & $638(84.8)$ & $308(48.3)$ \\
\hline \multicolumn{3}{|l|}{ Research type } \\
\hline Clinical science & $601(79.7)$ & $88(57.5)$ \\
\hline Basic science & $153(20.3)$ & $295(49.1)$ \\
\hline \multicolumn{3}{|l|}{ Subspecialty } \\
\hline Vascular & $138(18.3)$ & $65(47.1)$ \\
\hline Neuro-oncology & $184(24.4)$ & $115(62.5)$ \\
\hline Spine & $151(20.0)$ & $64(42.4)$ \\
\hline Functional & $61(8.1)$ & $31(50.8)$ \\
\hline Peripheral nerve & $13(1.7)$ & $6(46.2)$ \\
\hline Neurotrauma & $39(5.2)$ & $21(53.8)$ \\
\hline Pediatrics & 37 (4.9) & $21(56.8)$ \\
\hline Radiosurgery & $26(3.4)$ & $17(65.4)$ \\
\hline General & $105(13.9)$ & $43(41.0)$ \\
\hline \multicolumn{3}{|l|}{ Country of Origin } \\
\hline United States & $534(70.8)$ & $274(51.3)$ \\
\hline Canada & $31(4.1)$ & $19(61.3)$ \\
\hline Other & $189(25.1)$ & $90(47.6)$ \\
\hline \multicolumn{3}{|l|}{ Study Design } \\
\hline Randomized Control Trial & $17(2.3)$ & $10(58.8)$ \\
\hline Cohort Study & $381(50.5)$ & $193(50.7)$ \\
\hline Case-control study & $48(6.4)$ & $26(54.2)$ \\
\hline Case series & $125(16.6)$ & $52(41.6)$ \\
\hline Metanalysis & $7(0.9)$ & $5(71.4)$ \\
\hline \multicolumn{3}{|l|}{ Statistically Significant Results } \\
\hline Yes & $135(17.9)$ & $84(62.2)$ \\
\hline No & $619(82.1)$ & $299(48.3)$ \\
\hline \multicolumn{3}{|l|}{ New technology or technique } \\
\hline Yes & $278(36.9)$ & $146(52.5)$ \\
\hline No & $476(63.1)$ & $237(49.8)$ \\
\hline \multicolumn{3}{|l|}{ Principal author PhD } \\
\hline Yes & $158(21.0)$ & $91(57.6)$ \\
\hline No & $596(79.0)$ & $292(49.0)$ \\
\hline
\end{tabular}

research had a shorter median time to publication of 225 days compared to 509 days for clinical research. Industry sponsorship status was not readily apparent in the vast majority of abstracts and therefore was not collected. There was no difference in publication rate when comparing abstracts by country of origin, study design, presence of statistically significant results, mention of a new technology, technique or whether the principal author held a $\mathrm{PhD}$ (results not shown). 


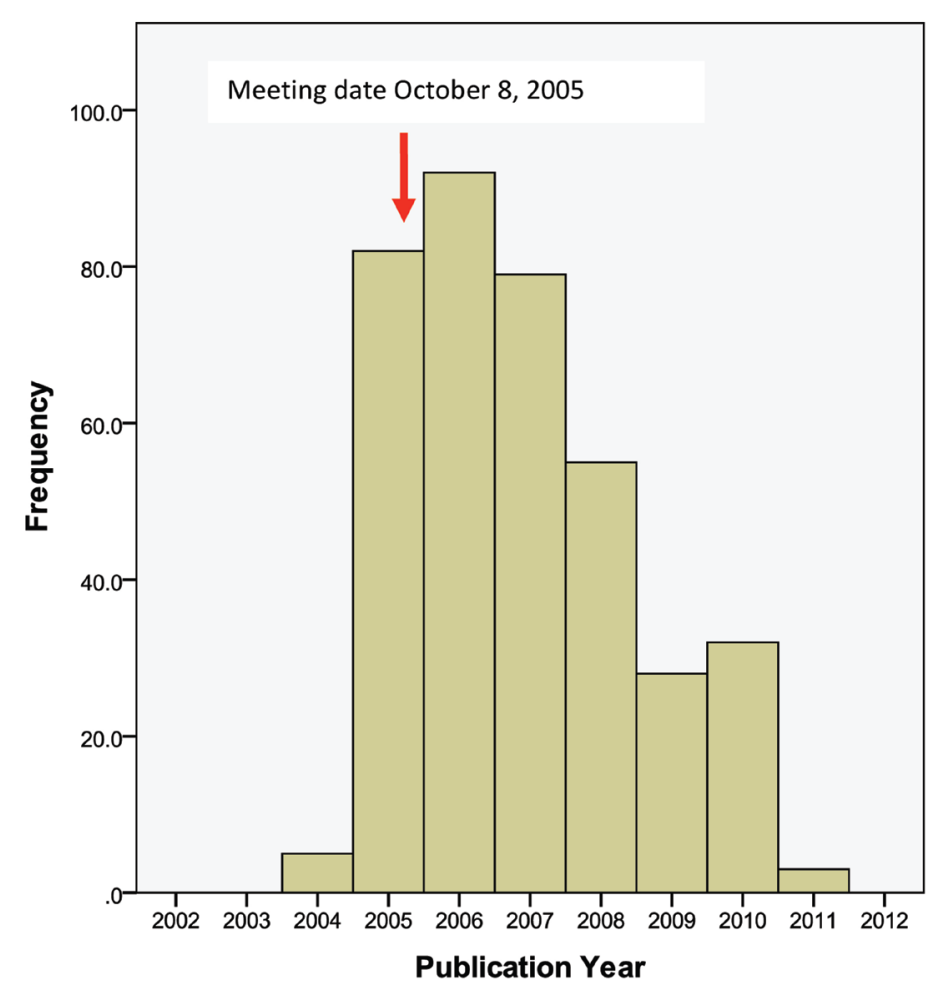

Figure 1. Year of publication for 383 abstracts that achieved full-text publication.

Table 2. Top Eight Journals of publication for 383 abstracts.

\begin{tabular}{ccc}
\hline Journal & Impact Factor (2010) & $n(\%)$ \\
Neurosurgery & 3.30 & $66(17.2)$ \\
Journal of Neurosurgery & 2.74 & $65(17.0)$ \\
Surgical Neurology & 1.26 & $17(4.4)$ \\
Journal of Neurosurgery Spine & 1.59 & $16(4.2)$ \\
Spine & 2.51 & $13(3.9)$ \\
Neurosurgical Focus & 2.35 & $12(3.1)$ \\
Acta Neurochirurgica & 1.33 & $8(2.1)$ \\
Cancer Research & 8.23 & 13.9
\end{tabular}

\subsection{Multivariate Predictors of Publication and Higher Impact Factor}

On multivariate binary logistic regression analysis neuro-oncology abstracts (OR 1.87; 95\% CI 1.31 - 2.67; $\mathrm{p}=$ 0.001) and those selected for oral presentations (OR 1.97; 95\% CI 1.30 - 2.99; $\mathrm{p}=0.001$ ) were each independent predictors of publication. Predictors of publication in journals with a higher absolute impact factor by multivariate linear regression analysis were neuro-oncology abstracts $(p=0.004)$, basic science research $(p=0.003)$ and abstracts selected for oral presentation $(p=0.036)$.

\section{Discussion}

We set out to determine the publication rate following abstract presentation at a major neurosurgical meeting. This is important because it is one generally accepted indicator of conference quality and provides insight into the completion process for research projects. We found that $50.8 \%$ of abstracts presented at the $55^{\text {th }}$ annual meeting of the CNS were published by 5.3 years of follow-up. The median time to publication was 450 days (15 
months) with a wide range (646 days prior to the conference to 1943 days following the conference). Abstracts selected for oral presentation and neuro-oncology abstracts were more likely to be ultimately published. Moreover, neuro-oncology abstracts, basic science research and abstracts selected for oral presentation were published in higher impact factor journals.

Our finding of a publication rate of $50.8 \%$ following abstract presentation compares favorably to the 2-year publication rate of $44.5 \%$ determined by Scherer and colleagues in a meta-analysis including 79 studies across a wide range of subspecialties including 29720 abstracts [4]. Moreover, of the publications that occurred following abstract presentation, 77\% occurred within 3 years of the congress. This finding is in keeping with publication trends in other fields such as orthopedic surgery which demonstrate that over $90 \%$ of publications occur within 4 years of the meeting presentation [4] [5] [11]. The median time to publication that we determined of 15 months is faster than the average for a wide variety of other subspecialties, in particular for the basic science research which has a median time to publication of 225 days [4]. This may reflect more dedicated research time allotted to clinicians participating in basic science research (although the proportion of basic science research carried out by a principal investigator with a $\mathrm{PhD}$ was no different than for clinical science) or a selection bias as a result of basic science work making up a minority of accepted abstracts (20\%).

Our calculated publication rate is higher than the publication rate determined in a recent report by Patel and colleagues which found a 5-year publication rate of 32.5\% for CNS and AANS annual meetings across three years [12]. In particular they found a publication rate of $29.8 \%$ for the 2005 CNS annual meeting. Some possible reasons to account for the higher publication rate in our study include that our publication search included 3 different databases as well as a crossover search in which abstracts that were initially found not to result in a publication would be searched a second time by a second team member in each database. This crossover search did yield to discovery of a significant number of additional publications (31/383; 8.1\%).

We found a pre-conference publication rate of $16 \%$, which is high compared to other fields such as anesthesia, and general surgery where the pre-congress publication rate is less than 5\% [7] [13] [14]. Accepted scientific etiquette would generally mandate a pre-congress publication be withdrawn or not submitted in the first place as presentation of an abstract would then be redundant. Perhaps our assumption that pre-congress publications and abstract presentations are identical in content may be false in some cases but this is not evident based on the level of detail available in the written abstracts.

Our finding that abstracts selected for oral presentation were more likely to be published has been previously described in other fields [4] [5] [10] [12] [15] [16]. Clearly the conference abstract selection process appears to identify those abstracts which are of high quality or address topics of current interest for oral presentation. This association is likely further bolstered by a low proportion of abstracts being selected for oral presentation (15\%) compared to meetings in other fields such as nephrology (48\%) and anesthesiology (45\%) [7] [10] as well as a competitive abstract selection process with only 754 of 1134 abstracts submitted (66\%) being accepted for presentation.

The association between neuro-oncology abstracts and publication was less expected. Interestingly, there was no difference in the proportion of principle investigators holding a $\mathrm{PhD}$ in neuro-oncology versus all other subspecialty areas nor was there any statistically significant difference in publication rate for principal investigators who held a PhD versus those who did not. Finally, our analysis did not reveal an increased publication rate associated with larger sample sizes, statistically significant results, country of origin or new surgical technique or technology. It is important to note that although statistically significant results were mentioned in $17.9 \%$ of abstracts, it was difficult to reliably determine whether these results were related to primary outcome due to a distinct lack of abstract detail in many cases.

Potential limitations of our study include that our publication search underestimate the true publication rate, as there may be publications in journals that are not indexed by the three databases that we selected. Data extraction was in some cases limited by a paucity of detail. Although standardization of abstract content may mitigate this problem strict abstract length parameters (e.g. 750 words for 2011 CNS annual meeting abstracts) may accentuate it. Our publication surveillance window was 5 years which was based on previous work demonstrating that greater than $90 \%$ of articles are published within 4 years of abstract presentation [4] [5]. This may have also contributed to an underestimation of the true publication rate. Our impact factor analysis was simplified to use 2010 impact factor values regardless of year of publication which may have impacted the results. Finally, by selecting the CNS annual meeting for our study we have examined abstracts that were predominantly from North America (75\%) which limits the generalizability of our results to other meetings based elsewhere. 


\section{Conclusion}

The publication rate following abstract presentation at a major neurosurgical meeting is $50.8 \%$ with a median time to publication of 450 days. Abstracts selected for oral presentation and neuro-oncology abstracts are more likely to be published. This publication rate compares favorably with a multi-disciplinary publication rate determined by meta-analysis of $44.5 \%$ and is an endorsement for conference quality [4].

\section{Disclosures}

The authors report no conflict of interest concerning the materials or methods used in this study or the findings specified in this paper.

\section{Contributions}

Author contributions to the study and manuscript preparation include the following. Conception and design: Elliott, Gourishankar, Mehta. Data acquisition: Elliott, Poon, Oliver. Analysis and interpretation of data: Elliott, Gourishankar. Manuscript preparation: Elliott. Critical revision: Gourishankar, Mehta. Study supervision: Gourishankar.

\section{References}

[1] Arrive, L., Dono, P., Lewin, M., Dahan, H., Monnier-Cholley, L. and Tubiana, J.M. (1996) Publication Rate of Original Papers Orally Presented at the Journees Fracaises de Radiologie. Journal of Radiology, 82, 1719-1722.

[2] Bhandari, M., Devereaux, P.J., Guyatt, G.H., Cook, D.J., Swiontkowski, M.F., Sprague, S. and Schemitsch E.H. (2002) An Observational Study of Orthopaedic Abstracts and Subsequent Full-Text Publications. The Journal of Bone and Joint Surgery (America Volume), 84, 615-621.

[3] Herbison, P. (2004) Full Publication of Abstracts of Randomized Controlled Trials Published at International Continence Society Meetings. Neurourology and Urodynamics, 23, 101-103. http://dx.doi.org/10.1002/nau.20003

[4] Scherer, R.W., Dickersin, K. and Langenberg, P. (1994) Full Publication of Results Initially Presented in Abstracts. A Meta-Analysis. JAMA, 272, 158-162. http://dx.doi.org/10.1001/jama.1994.03520020084025

[5] Scherer, R.W., Langenberg, P. and von Elm, E. (2007) Full Publication of Results Initially Presented in Abstracts. Cochrane Database of Systematic Reviews, 2, Article No.: MR000005. http://dx.doi.org/10.1002/14651858.mr000005.pub3

[6] Carrol, A.E., So, C.M., Tarini, B.A., Ringold, S. and Chirstakes, D.A. (2003) Does Presentation Format at the Pediatric Academic Societies Annual Meeting Predict Subsequent Publication? Pediatrics, 112, 1238-1241.

[7] Castillo, J., Garcia-Guasch, R. and Cifuentes, I. (2002) Fate of Abstracts from the Paris 1995 European Society of Anaesthesiologists Meeting. European Journal of Anaesthesiology, 19, 888-893. http://dx.doi.org/10.1097/00003643-200212000-00006

[8] De Bellefeuille, C., Morrison, C.A. and Tannock, I.F. (1992) The Fate of Abstracts Submitted to a Cancer Meeting: Factors Which Influence Presentation and Subsequent Publication. Annals of Oncology, 3, 187-191.

[9] Dumville, J.C., Petherick, E.S. and Cullum, N. (2005) When Will I See You Again? The Fate of Research Findings from International Wound Care Conferences. International Wound Journal, 5, 26-33. http://dx.doi.org/10.1111/j.1742-481X.2007.00343.x

[10] Glick, N., MacDonald, I., Knoll, G., Brabant, A. and Gourishankar, S. (2006) Factors Associated with Publication Following Presentation at a Transplantation Meeting. American Journal of Transplantation, 6, 552-556. http://dx.doi.org/10.1111/j.1600-6143.2005.01203.x

[11] Daluiski, A., Kuhns, C.A., Jackson, K.R. and Lieberman, J.R. (1998) Publication Rate of Abstracts Presented at the Annual Meeting of the Orthopaedic Research Society. Journal of Orthopaedic Research, 16, 645-649. http://dx.doi.org/10.1002/jor.1100160603

[12] Patel, A.J., Cherian, J., Fox, B.D., Whitehead, W.E., Curry, D.J., Luerssen, T.G. and Jea, A. (2011) Publication Patterns of Oral and Poster Presentations at the Annual Meetings of the Congress of Neurological Surgeons and the American Association of Neurological Surgeons. Journal of Neurosurgery, 115, 1258-1261.

[13] Gavazza, J.B., Foulkes, G.D. and Meals, R.A. (1996) Publication Pattern of Papers Presented at the American Society for Surgery of the Hand Annual Meeting. The Journal of Hand Surgery, 21A, 742-745.

http://dx.doi.org/10.1016/S0363-5023(96)80185-7 
[14] Hamlet, B.W.P., Fletcher, A. and Meals, R.A. (1997) Publication Patterns of Papers Presented at the Annual Meeting of the American Academy of Orthopedic Surgeons. The Journal of Bone and Joint Surgery (America Volume), 79, 1138-1143.

[15] Krzyzanowska, M.K., Pintilie, M. and Tannock, I.F. (2003) Factors Associated with Failure to Publish Large Randomized Trials Presented at an Oncology Meeting. JAMA, 290, 495-501.

[16] Wang, J.C., Yoo, S. and Delamarter, R.B. (1999) The Publication Rates of Presentations at Major Spine Specialty Society Meetings. Spine, 24, 425-427. http://dx.doi.org/10.1097/00007632-199903010-00002 